\section{Psoas muscle tuberculosis in Greece: report of two cases}

\author{
Leonidas Grigorakos, ${ }^{1,2}$ \\ Vassilis Sgountzos, ${ }^{3}$ \\ Sophia Simopoulou, ${ }^{4}$ Daria Lazarescu, \\ Domenica Mavropanou, ${ }^{2}$ \\ Anthi Georgiadou, ${ }^{2}$ Vassilis Tamvakis ${ }^{3}$ \\ ${ }^{1}$ Faculty of Nursing, National and \\ Kapodistrian University of Athens, \\ Athens; ${ }^{2}$ Intensive Care Unit, Trauma \\ Hospital of Athens, Kifissia; ${ }^{3}$ Sotiria \\ Regional Chest Diseases Hospital, \\ Athens; ${ }^{4}$ Peripheral Anticancer \\ Oncology Hospital of Athens \\ "Agios Savvas", Athens, Greece
}

\begin{abstract}
Between January 2013 and January 2014, two patients with lower left abdominal pain were admitted to Sotiria Chest Diseases Hospital, Athens, Greece. They were diagnosed with psoas muscle tuberculosis based on the results of clinical evaluation, laboratory tests and radiological findings from chest X-rays and computed tomography. Administration of antitubercular chemotherapy reduced the inflammation, while clinical symptoms significantly improved within the first three weeks. Patients were discharged from the hospital two months later. Their follow-up consists of monthly check-ups, while continuing the anti-tuberculosis treatment successfully. We underline the importance of keeping a high suspicion of muscle tuberculosis in patients with psoas abscess, which, although not a seldom phenomenon, it should be taken into consideration as an etiology of muscular disease not only in regions where tuberculosis is endemic but also in countries with high incidence of immigrants or refugees originating from such areas.
\end{abstract}

\section{Introduction}

Tuberculosis (TB) is one of the most serious infectious diseases and it still represents a considerable public health problem of the $21^{\text {st }}$ century due to its high risk of person-to-person transmission, morbidity, and mortality. ${ }^{1}$ Thus, early diagnosis followed by adequate treatment is essential to prevent its consequences. Extra-pulmonary involvement emerges in approximately $20 \%$ of all cases of tuberculosis in immunocompetent patients $[50 \%$ in human immunodeficiency virus (HIV)-positive individuals] of which about one tenth involve the musculoskeletal system, mostly spondylitis, osteomyelitis or arthritis. ${ }^{2,3}$ However, involvement of skeletal muscle without co-existing active disease is very rare, with incidence of primary muscular tuberculosis being reported as $0.015 \%$ of all cases. ${ }^{4-6}$

Muscular tuberculosis occurs as a contagious spread from neighboring tuberculous focus in the bones or joints, through the rupture and erosion of the walls of an active peripheral tuberculous cavity (i.e. from cold paravertebral abscess to the psoas or abdominal muscles). ${ }^{1,7}$ The fact that it is very rarely detected in skeletal muscles is attributed to tissues' resistance to mycobacteria because of: i) poor $\mathrm{O}_{2}$ in the muscle tissue; ii) scarcity of reticulo-epithelioid tissue; and iii) presence of lactic acid within skeletal muscle. ${ }^{4}$ Actually, the primary type of disease can only be detected in case these have been transplanted to a muscle through an infected needle while performing a thoracentesis or an intramuscular injection. ${ }^{8}$ Moreover, quite often muscle tuberculosis is misdiagnosed as sarcoma, soft tissue tumor, fungal infection, parasitic infectious hydatid cyst, lipoma or hematoma. ${ }^{9}$ This potential misdiagnosis is the main reason to present our experience with two such cases.

\section{Case Report}

Two patients originating from African countries were admitted to the Sotiria Chest Diseases Hospital between January 2013 and January 2014 with lower left abdominal pain for an undefined period of time (Table 1). Health care personnel could not receive a clear medical history since barriers of communication with patients were high due to their lack of knowledge of both Greek and English language.

At arrival, medical examination did not reveal symptoms of fever. However, they had intense cough and looked thin. Laboratory examination, sputum examination for Koch's bacillus (5 samples) and chest X-rays did not show pathological findings in neither of patients. Their tuberculin skin test was positive, with the induration measuring 30 and $22 \mathrm{~mm}$, respectively.

Both patients' clinical images, combined with findings of computed tomography (CT) that revealed abscesses at the left psoas muscle, were suggestive of muscle tuberculosis while no intra-articular extension was observed.

The study was conducted according to the principles of the Declaration of Helsinki, while informed consent was obtained from both patients.

Psoas abscess was aspirated by percutaneous CT-guided drainage in both patients.
Correspondence: Leonidas Grigorakos, Faculty of Nursing, National and Kapodistrian University of Athens, 2 Nikis Street, 14561, Kifissia, Athens, Greece.

Tel: +30.210.3709522 - Fax: +30.210.3709520.

E-mail: grigorakos@parliament.gr

Key words: Muscle tuberculosis; Psoas muscle abscess; Greece.

Contributions: all authors substantially contributed to the conception and design of the study, acquisition, analysis and interpretation of data, and drafting of the article. All authors approved the final version to be published.

Conflict of interest: the authors declare no potential conflict of interest.

Received for publication: 1 February 2016.

Revision received: 31 May 2016.

Accepted for publication: 28 June 2016.

This work is licensed under a Creative Commons Attribution NonCommercial 4.0 License (CC BY-NC 4.0).

(C) Copyright L. Grigorakos et al., 2017

Licensee PAGEPress, Italy

Chest Disease Reports 2017; 5:5777

doi:10.4081/cdr.2017.5777

About 15 and $14 \mathrm{~mL}\left(1^{\text {st }}\right.$ and $2^{\text {nd }}$ patient, respectively) of grayish white pus were drained and sent for microbiological investigations and histopathology. Gram stain showed moderate pus cells but no bacteria. Aerobic culture was sterile. Ziehl Neelsen's staining was positive for acid-fast bacilli (1 to 3 bacilli could be seen in the whole smear in the case of both patients). The diagnosis was then confirmed for both patients with positive polymerase chain reaction (PCR) for Mycobacterium tuberculosis. Patients were diagnosed with tubercular psoas abscess based on clinical history, intraoperative finding of abscess in psoas muscle, tuberculin test, acid-fast bacilli on pus drain, and positive PCR. They were subjected to anti-tuberculosis therapy in our clinic, receiving for two months daily per os dosages of: i) $300 \mathrm{mg}$ isoniazid, and $600 \mathrm{mg}$ rifampicin; ii) $1250 \mathrm{mg}$ of ethambutol in the form of dexambutol tablets; iii) $1500 \mathrm{mg}$ pyrazinamide; and iv) $25 \mathrm{mg}$ pyridoxine. Within a time-span of three weeks both patients had positive clinical improvement (they felt better and their appetite improved). After having assessed their socioeconomic ability and not being sure that they would be able to follow the accurate medication outside the hospital, patients remained hospitalized for two months. Then, they were released in good health and continued their treatment with 
Table 1. Patients' characteristics.

\begin{tabular}{lcc}
\hline Patients' characteristics & 1st patient $^{\text {nd }}$ patient \\
Age (years) & 31 & 28 \\
Sex & Male & Female \\
\hline Weight $(\mathrm{kg})$ & 61 & 54 \\
Length $(\mathrm{cm})$ & 172 & 168 \\
\hline Symptoms at admission & Lower left abdominal pain & Lower left abdominal pain \\
Tuberculin skin test $(\mathrm{mm})$ & 30 & 22 \\
\hline
\end{tabular}

isoniazid and rifampicin for seven months. After percutaneous CT-guided drainage and 7 months of antimycobacterial therapy, contrast CT revealed no evidence of psoas abscess. Both patients come for monthly check-ups at the Anti-tuberculosis Department of our Hospital.

\section{Discussion}

About half of the cases of musculoskeletal TB have evidence of active or healed pulmonary TB with a single site of involvement. ${ }^{8}$ However, multiple locations are not uncommon and occur more often in patients who are immunocompromised, including HIV-infected patients. ${ }^{7}, 10$ On the contrary, primary tuberculous psoas abscess in a patient without any apparent focus and without any previous predisposing pathology is an extremely rare process. ${ }^{11}$ Even though literature describes lumbar pain, limitation of hip mobility and fever as the classic triad of symptoms in patients with tuberculous psoas abscess, patients arrived at our hospital only with lower left abdominal pain. ${ }^{11}$ Primary muscle tuberculosis of the psoas requires a multidisciplinary management and differential diagnosis. During the diagnostic stage of the tuberculous abscess it is important to look for any possible focus of infection: lung, spine, gastrointestinal tract and genitourinary system. Only once these foci have been ruled out one can talk about a primary abscess. ${ }^{12,13}$ More so, as researchers report, lack of caseation or acid-fast bacilli in the culture of the biopsy samples do not exclude muscle tuberculosis. ${ }^{14}$ At this point, the findings of CT scans of the involved muscle as well as microbiological and histological examinations are very helpful in differential diagnosis. A CT usually demonstrates findings suggestive of muscle tuberculosis, rather than pyogenic infections or malignancy. These include mediastinal lymphadenopathy with low central density in patients with chest wall muscle tuberculosis, and the absence of adjacent venous thrombosis or cellulitis (which usually occurs in pyogenic myositis) in patients with muscle tuberculosis of the extremities. ${ }^{6,15}$

\section{Conclusions}

Even though not a seldom phenomenon, muscle tuberculosis should be taken into consideration as an etiology of muscular disease not only in regions where tuberculosis is endemic but also in countries with high incidence of immigrants or refugees originating from such areas. A definite diagnosis of psoas muscle tuberculosis should follow a clinically-directed multidisciplinary approach based on microbiological and histological examination.

\section{References}

1. Al-Tawfiq JA. Multifocal systemic tuberculosis: the many faces of an old nemesis. Med Sci Monit 2007;13:5660.

2. Simon S, Fodor D, Valasciuc R, et al. A rare case of primary tuberculous pyomyositis. Case report. Med Ultrason 2011;13:245-8

3. Yao DC, Sartoris DJ. Musculoskeletal tuberculosis. Radiol Clin N Am
1995;33:679-89

4. Kulkarni SA, Patil SS, Kulkarni P, et al. Primary tuberculous myositis: a rare clinical entity. Indian J Tuberc 2013; 60:241-4.

5. Alawad AAM, Gismalla MD Tuberculous abscess of the anterior abdominal wall: an unusual site of presentation. Global J Med Clin Case Rep 2015;2:8-9.

6. Grigorakos L, Sgountzos V, Lazarescu D, et al. Primary thoracic muscle tuberculosis: two case reports. J Med Case Rep 2016;10:229.

7. Wang JY, Lee LN, Hsueh PR, et al. Tuberculous myositis: a rare but existing clinical entity. Rheumatology 2003;42:836-40.

8. Dhakal AK, Shah SC, Shrestha D, et al. Tuberculosis presenting as multiple intramuscular nodules in a child: a case report. J Med Case Rep 2015;9:72.

9. Bhatty SM, Prakash JS, John B. Pulmonary tuberculous abscess of vastus lateralis muscle. JK Science 2011; 13:37-8.

10. Agarwal RP, Mohan N, Gary RK, et al. Clinicosocial aspect of osteo-articular tuberculosis. J Indian Med Assoc 1990; 88:307-9.

11. Berge M, de Marie S, Kuipers T, et al. Psoas abscess: report of a series and review of the literature. Neth $\mathrm{J}$ Med 2005;63:413-6.

12. Cano C, Alconada RG, Borobio G, et al. A rare cause of hip pain: primary tuberculous psoas abscess. Case report. JSM Clin Case Rep 2015;3:1078.

13. Chawla K, D'Souza A, Bhat S, et al. Primary tubercular psoas abscess: a rare presentation. J Infect Dev Ctries 2012; 6:86-8.

14. Kobayashi H, Kotoura Y, Hosono M, et al. Solitary muscular involvement by tuberculosis: CT, MRI, and scintigraphic features. Comput Med Imag Grap 1995;19:237-40.

15. Batra S, Ab Naell M, Barwick C, et al. Tuberculous pyomyositis of the thigh masquerading as malignancy with concomitant tuberculous flexor tenosynovitis and dactylitis of the hand. Singapore Med J 2007;48:1042-6. 